Proceeding of the $1^{\text {st }}$ ICEENG conference, 24-26 March, 1998.

$A C 1-\angle 50$

MIITIRY TECHNICAI COLLEGE ICEEYG g8
CAIRO-EGYPT

\title{
NEW MESSAGE AUTHENTICATION CODE USING UNIMODAL CHAOTIC MAPPING
}

\author{
Alaa Fahmy*
}

\section{ABSTRACT}

Message authentication is a procedure established between two ends, (sender and receiver), allows each end to verify the integrity of the received message. The contents of a message are authenticated by verifying the correctness of message authentication code (MAC), which is computed by the sender and is appended to the message. In this paper, we present a new method to generate the MAC, using unimodal chaotic mapping.

\section{INTRODUCTION}

The unpredictability of chaotic dynamical systems arises from the fact that small error in the initial conditions corresponds to different change in behavior, i.e., chaotic dynamical systems are sensitive dependence on initial conditions [1]. When the system is allowed to evolve, it loses all the information about the initial condition therefore, we used this property to generate the message authentication code.

\section{MESSAGE AUTHENTICATION CODE GENERATION}

The unimodal map given by eq.(1) has been used to generate the MAC.

$X_{n+1}=\mu \cdot X_{n} \cdot\left(1-X_{n}\right)$

"Ph.D., Military Technical College. 
Proceeding of the $1^{\text {st }}$ ICEENG conference, 24-26 March, 1998.

The Message authentication can be constructed as follows:

(i) Divide the encoded plaintext, $\mathrm{Z}$, into $\mathrm{N}$ blocks, $\left\{\mathrm{Z}_{\mathrm{i}}\right\}_{\mathrm{i}=1: \mathrm{N}}$, of fixed size $\mathrm{b}$ (56 bits). Pad Z if necessary.

(ii) Use the transformation $\mathrm{F}: \mathrm{Z}_{\mathrm{i}} \rightarrow \mathrm{R}_{\mathrm{i}}$ to transform the binary block, $\mathrm{Z}_{\mathrm{i}}$, into

decimal number $R_{i} \in(0,1)$, and assign $R_{i}$ to an initial condition $x_{0 i}$.

(iii) Iterate eq.(1) with an initial condition $\mathrm{x}_{\mathrm{oi}}$ and with $\mu \geq 3.9$ (chaotic region). The number of iterations $\mathrm{k}$ is set to 300 iterations. Only the last $\mathrm{b}$ iterations are taken and

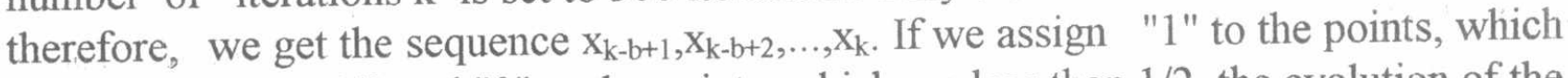
are greater than $1 / 2$ and " 0 " to the points, which are less than $1 / 2$, the evolution of the logistic map will generate, from every initial condition $\left(\mathrm{x}_{\mathrm{oi}}\right)$, a sequence $\left(\mathrm{Y}_{\mathrm{i}}\right)$ of $1 \mathrm{~s}$ and $0 \mathrm{~s}$, which resemble the tossing of a coin.

(iv) Repeat the steps (ii-iii) $\mathrm{N}$ times and exclusive-OR the blocks of the output sequence $Y_{i}$, we get the function $B(M)=Y_{1} \oplus Y_{2} \oplus \ldots \oplus Y_{N}$. The function $B$ transforms a plaintext $\mathrm{M}$ of arbitrary length into a relatively short, fixed length (56 bits), pattern of bits $B(M)$, with a high probability that $B(M)$ will differ from $B\left(M^{\prime}\right)$ whenever the recovered plaintext $\left(\mathrm{M}^{\prime}\right)$ different from the original plaintext $(\mathrm{M})$.

(v) Encipher the function $B(\mathrm{M})$, using the cryptographic algorithm presented in [2]. The enciphered value of $B(M)$ represents the MAC.

At the other end (receiver) the same procedures are applied to reproduce the message authentication code. The receiver compares the calculated message authentication code with the received one. If they are equal, the message is accepted (genuine message) otherwise, it is rejected (forge message).

\section{RESULTS AND DISCUSSIONS}

The unimodal map, given by eq.(1), can be regarded as a one-way function. That is, given an initial condition $x_{0}$, it is feasible to calculate $x_{n+1}$, but it is computationally infeasible to calculate $\mathrm{x}_{0}$ given $\mathrm{x}_{\mathrm{n}+1}$. Since the construction of the 
MAC is based on such function, it is computationally infeasible to generate two messages having the same MAC, or to produce any message having a prescribed MAC.

The number of operations required for such procedures is of the order of $2^{56}$ operations. Whilst the MAC derived from the data encryption standard (DES) algorithm, using either cipher block chaining mode or cipher feedback mode, has $2^{24}$ and $2^{32}$ operations for telecommunication and financial applications respectively [3]. The constructed MAC is simple and it is convenient whenever the cryptographic algorithm is available either in software or in hardware. In contrast, the MAC generated from the DES is convenient only if a hardware DES function is available because the algorithm is slow and inconvenient in software. In addition, a single error in the ciphertext causes a single error in the recovered plaintext, and a drastic change in the message authentication code.

The cryptosystem presented in [2], which is based on stream cipher idea can be used to illustrate the error propagation and key-ciphertext avalanche effect. Figure 1 illustrates the error propagation property. Changing one bit in the ciphertext causes a drastic change in the message authentication code. It is also clear from Fig.2 that small change in the initial conditions, with fixed piaintext, will result to radical change in both the ciphertext and the MAC.

Therefore, we can use this idea of MAC generation with the conventional cryptographic systems, based on stream cipher idea, for both data transmission and authentication applications. Figure 3 illustrates error propagation and self synchronization (single bit change in the ciphertext causes a single bit change in the recovered plaintext) property when enciphering both the plaintext and the generated MAC with the cryptographic algorithm presented in [2]. 
Proceeding of the $1^{\text {st }}$ ICEENG conference, 24-26 March, 1998.

$A C 1-459$

\section{CONCLUSION}

A new method to generate the message authentication code is introduced. It is based on the sensitive dependence on initial conditions of the chaotic systems. In this way, any single bit change in the ciphertext results in a drastic change in the message authentication code. It can be used with cryptographic algorithms, based on stream cipher idea for both data transmission and authentication applications.

\section{REFERENCES}

[1] Hao Bai-Lin, Chaos II.

[2] Sobhy, M.I. and Fahmy, A., "Cryptographic Algorithm Based on Chaotic Behavior", Tenth National Radio Science Conference, Cairo, Egypt, Feb. 16$18,1993$.

[3] National Bureau of Standards, "DES Modes of Operation," FIPS Publication 81, Dec. 1980. 
Proceeding of the $1^{\text {st }}$ ICEENG conference, 24-26 March, 1998.

$A C 1-400$

\title{
Ciphertext C
}
A 34 B 22 D 08 C F 00 F
B 7 B 487809D D 0 C B

Ciphertext $C^{\prime}$
A 24 B 22D 08 C F 00 F
B 7 B 487809 D D 0 C B

MAC

D 3 D F A 6

MAC ${ }^{\prime}$

\author{
64 D E F 6
}

Fig.1 MAC For Single Bit Change in Ciphertext 
Proceeding of the $1^{\text {st }}$ ICEENG conference, 24-26 March, 1998.

$A C 1-461$

Message (Plaintext) M

\section{HELLO HELLO}

\section{Plaintext in Hexadecimal Form}

$$
\begin{aligned}
& 48454 \mathrm{C} 4 \mathrm{C} 4 \mathrm{~F} 2048 \\
& 454 \mathrm{C} 4 \mathrm{C} 4 \mathrm{FD} 3 \mathrm{DFA} 6
\end{aligned}
$$

Ciphertext C For $\mathrm{X}_{0}=\mathbf{0 . 1 2 6 8 5 7 0 4 3 9}$
A 34 B 22D 08 C F 00 F B 7 B 487809 D D 0 C B

MAC

$$
64 \text { D E F } 6
$$

Ciphertext $C^{\prime}$ For $X_{0}^{\prime}=0.1268570434$

$$
\begin{aligned}
& \text { A C } 932 \text { F C A 2 C B E } 68 \\
& 538 \text { B } 2 \text { F } 68 \text { A E } 941 \text { A }
\end{aligned}
$$

MAC

$$
\text { E } 09 \text { B } 77
$$

Fig.2 Key-Ciphetext Avalanche Effect 
Proceeding of the $1^{\text {st }}$ ICEENG conference, 24-26 March, 1998.

Message M

\section{HELLO HELLO}

Ciphertext C
A 34 B 22 D08 C F 00 F
B 7 B 487809 D D 0 C B

MAC
D 3 D F A 6

Ciphertext $C^{\prime}$
A 24 B 22 D 08 C F 00 F
B 7 B 487809 D D 0 C B

Recovered Plaintext M'

\section{IELLO HELLO}

MAC $^{\prime}$

$$
64 \text { D E F } 6
$$

Fig.3 Self Synchronization and Error

Propagation Property 